treatment than in patients with toxicity of grade $0-1$, and $t_{\max }>30$ min was associated with gastrointestinal and hematologic toxicity. No association was found between DPD activity and 5-FU toxicity.

The authors conclude that analysis of 5-FU and 5-FDHU pharmacokinetics before treatment would be helpful in identifying patients at risk from 5-FU therapy, and that pharmacokinetic screening for 5-FU and 5-FDHU disposition is necessary to elucidate the relationship between DPD and 5-FU catabolism.

Original article Bocci G et al. (2006) A pharmacokineticbased test to prevent severe 5-fluorouracil toxicity. Clin Pharm Ther 80: 384-395

\section{Detection of curable stage I lung cancer by annual CT screening}

Annual CT screening with spiral CT is a valuable method for diagnosing stage I lung cancer; however, little is known about the outcome of CT screening in populations at risk for lung cancer. Recently, the results of a large collaborative study suggested that annual CT scanning can detect clinical stage I lung cancer, at which point cure by surgery is highly possible.

In this study, conducted from 1993 to 2005, 31,567 asymptomatic persons aged 40 years or older, who were at risk for lung cancer owing to various reasons (i.e. history of smoking, exposure to second-hand smoke or occupational exposure to asbestos, beryllium, uranium or radon) were screened using low-dose CT. Of these participants, 27,456 were re-screened 7-18 months after the initial screening. Following baseline and annual screenings, 484 participants were diagnosed with lung cancer. The estimated 10-year survival rate for these patients, regardless of the stage of the disease or treatment, was $80 \%(95 \% \mathrm{Cl} 74-85 \%)$. A subgroup of 412 patients was diagnosed with clinical stage I lung cancer and the estimated 10 -year survival rate of this group was $88 \%$ (95\% Cl 88-91\%). The estimated 10-year survival rate of 302 patients with stage I lung cancer who underwent surgical resection within 1 month following diagnosis was 92\% (95\% Cl 88-95\%), while all untreated patients $(n=8)$ died within 5 years of diagnosis.

The authors conclude that the detection of curable lung cancer by CT screening in populations at risk for this disease could avert approximately $80 \%$ of deaths from this type of cancer.

Original article Henschke CI et al. (2006) Survival of patients with stage I lung cancer detected on CT screening. N Engl J Med 355: 1763-1771

\section{Previously unrecognized genetic mutations in colorectal and breast cancers}

Few genome-wide studies have been performed to identify cancer-related mutations - most have been identified by analysis of a few genes, or from studies in cancer-prone families. Sjöblom and colleagues analyzed sequence data from 13,000 protein-encoding genes in 11 breast and 11 colorectal cancers. Previously uncharacterized mutations were present in 1,149 genes, which were reanalyzed in a validation study of 24 additional tumors. In total, the authors identified 921 confirmed somatic mutations in breast cancers and 751 in colorectal cancers.

Analysis revealed that 189 of the 1,149 genes probably had an elevated mutation frequency (122 genes in breast and 69 in colorectal cancers). Breast and colorectal cancers harbored $\sim 12$ and $\sim 9$ mutations, respectively, in these 189 candidate cancer genes. By contrast, the authors estimated that these cancers harbored mutations in 81 and 105 protein-encoding genes, which suggests that more mutations occur during tumorigenesis than previously thought.

Intriguingly, colorectal cancer mutations were predominantly transitions (i.e. $\mathrm{C}>\mathrm{G}$ or $\mathrm{T}>\mathrm{A}$ substitutions); a similar bias has been observed for CpG-island mutations in TP53 (which encodes the tumor-suppressor p53), but the authors suggest that this phenomenon is genome-wide. The mutation profiles of breast and colorectal cancers differed: metalloproteinase genes were more frequently mutated in colorectal than in breast tumors, whereas genes for transcriptional regulators were mutated in both cancer types, although the specific genes affected varied with tumor type.

The authors speculate that candidate cancer genes belong to a limited number of molecular pathways. They say that their results provide many promising avenues for future research.

Original article Sjöblom T et al. (2006) The consensus coding sequences of human breast and colorectal cancers. Science 314: 268-274 